\title{
Radiation shielding for underground low-background experiments
}

\author{
D.Y. Stewart* , P.F. Harrison, B. Morgan, Y. Ramachers \\ University of Warwick, Coventry, CV4 7AL, UK
}

\begin{abstract}
The design task of creating an efficient radiation shield for the new COBRA doublebeta decay experiment led to a comprehensive study of commercially available shielding materials. The aim was to find the most efficient combination of materials under the constraints of an extreme low-background experiment operating in a typical underground laboratory. All existing shield configurations for this type of experiment have been found to perform sub-optimally in comparison to the class of multilayered configurations proposed in this study. The method used here to create a specific shield configuration should yield a close to optimal result when applied to any experiment utilising a radiation shield. In particular, the survey of single material response to a given radiation source turns out to give a guideline for the construction of efficient multilayer shields.
\end{abstract}

Key words: double-beta decay, radiation shielding, Monte-Carlo, dark matter, low background

PACS: 23.40.-s, 24.10.Lx, 28.20.Gd

\section{Introduction}

The study of radiation shielding has a large interdisciplinary scope, ranging from high-energy physics accelerator infrastructure to medical physics and engineering science [2]. For generic low-background experiments utilising radiation detectors of any kind, a radiation shield is a major part of the set-up [3]. Rare event searches, as in particle dark matter and double-beta decay search experiments, rely heavily upon an extremely low level of background contributions from environmental radiation sources. So too do experiments

\footnotetext{
* Corrresponding author.

Email address: d.y.stewart@warwick.ac.uk (D.Y. Stewart).
} 
measuring tiny radioactive contaminations of samples, e.g. in archaeology and tracer searches in oceanography.

In the framework of the authors' main research project, the COBRA doublebeta decay experiment [1], the focus was on the design of a new radiation shield as one of the major constructional parts for the success of the current R\&D efforts into COBRA. A survey of existing shields for similar experiments soon showed that hardly any details are published, although they always constitute a major part of the physics programme. Therefore, it was decided that the issue should be investigated independently to see whether it would be viable to improve on existing designs, not only for the specific case of the COBRA experiment but in general.

The following strategy has been implemented for this study:

- Constrain the database of viable shielding materials to commercially available and mechanically convenient samples. A typical radiation shield weighs in excess of several hundred kilograms, hence cost and construction are important factors.

- Examine each single material for its radiation interaction properties for all relevant radiation sources. In our case, these consist of $\gamma$-radiation and neutrons. It should be pointed out that this study concentrates on neutron radiation and that $\gamma$-radiation mainly becomes a concern when originating from inelastic neutron reactions, rather than in isolation. In underground laboratories, the hadronic component of cosmic rays is absent and for the deeper laboratories, the muonic component is suppressed compared to environmental radioactivity [3].

- Maximise the attenuation for a specific radiation source by combinations of suitable materials, building a multilayer shield. The motivation for this process originates from general characteristics of shielding, i.e. (a) scatter particles to lower energies and (b) absorb particles, where (b) is always a stronger effect at lower energies. However, this rather simple few-parameter problem is complicated by the fact that particle number is generally not conserved for radiation interaction in matter. Build-up factors [4] are important and render the whole problem tractable only numerically, i.e. using Monte-Carlo simulations.

- Maximise the attenuation for any combination of radiation sources. It is understood that this might not always be necessary for every experiment, especially those that are insensitive to certain sources due to strong discrimination capabilities, as in dark matter experiments (see for instance $[5])$.

This text then concludes with a comparison of our proposed multilayer shields with 'standard' shields, employed in existing underground experiments. 
Table 1

Shielding materials and parameters used in the simulations. Note that mixtures are typically given as weight percent, whereas an atom number ratio is more useful for specifying materials for simulations. Natural isotope composition is always assumed.

\begin{tabular}{|c|c|c|}
\hline Material & Composition [atom number ratio] & Density $\left[\mathrm{g} / \mathrm{cm}^{3}\right]$ \\
\hline Lead & nat. & 11.34 \\
\hline Iron & nat. & 8.96 \\
\hline Copper & nat. & 7.87 \\
\hline $\mathrm{PE}$ & $\mathrm{CH}_{2}[1: 2]$ & 0.92 \\
\hline $\mathrm{PE}-\mathrm{Bi}^{1}$ & $\mathrm{CH}_{2}, \mathrm{Bi}[1: 2: 0.14]$ & 3.0 \\
\hline $\mathrm{PE}-\mathrm{B} 30 \%{ }^{1}$ & $\mathrm{CH}_{2}, \mathrm{~B}[1: 2: 0.6739]$ & 1.12 \\
\hline $\mathrm{PE}-\mathrm{B} 5 \% 1$ & $\mathrm{CH}_{2}, \mathrm{~B}[1: 2: 0.0788]$ & 0.95 \\
\hline $\mathrm{PE}-\mathrm{Li}^{1}$ & $\mathrm{CH}_{2}, \mathrm{Li}[1: 2: 0.1231]$ & 1.06 \\
\hline Premadex ${ }^{2}$ & HCOLi [1:0.35:0.2217:0.0162] & 1.0 \\
\hline Water & $\mathrm{H}_{2} \mathrm{O}[2: 1]$ & 1.0 \\
\hline
\end{tabular}

${ }^{1}$ Thermo-Electron Corporation; ${ }^{2}$ Wardray Premise Group

\section{Single Materials Survey}

The motivation to focus this study on neutron sources is twofold. First, studies and data on the subject of $\gamma$-radiation on various shielding materials exist (see [3], [4], [6] and references therein), even with the scope of examining multilayer shields [7]. Second, the COBRA experiment, utilising CdZnTe semiconductor detectors will be particularly sensitive to thermal neutron capture reactions on ${ }^{113} \mathrm{Cd}$, producing $\gamma$-radiation in the relevant signal energy region (above two $\mathrm{MeV}$ ).

Several recent studies for underground experiments quantified design criteria for shielding; in particular neutron shielding was targeted due to interest in new dark matter search experiments $[8,9,10]$. However, none of those experiments aimed at a complete description i.e. including $(n, \gamma)$-reactions and shielding effects on the combined neutron and photon population.

For this study, commercially available shielding materials were selected, as summarised in Table 1. Most materials tabulated here are specialised neutron shielding materials (the hydrocarbon class) but included are a few pure metals. As a representation of typical neutron fluxes as function of energy in a deep underground laboratory, the measured neutron flux from Gran Sasso was adopted [11] (see also the updated discussion about Gran Sasso neutron fluxes [12]), see Table 2. 
Table 2

Measured neutron flux as function of the indicated energy bins, from [11].

\begin{tabular}{ll} 
Energy bin $[\mathrm{MeV}]$ & $\begin{array}{l}\text { n-flux } \\
\left(10^{-6} \mathrm{~cm}^{-2} \mathrm{~s}^{-1}\right)\end{array}$ \\
\hline$<50 \times 10^{-9}$ & $1.07 \pm 0.05$ \\
$50 \times 10^{-9}-10^{-3}$ & $1.99 \pm 0.05$ \\
$10^{-3}-2.5$ & $0.53 \pm 0.08$ \\
$2.5-5$ & $0.18 \pm 0.04$ \\
$5-10$ & $0.04 \pm 0.01$ \\
$10-15$ & $(0.7 \pm 0.2) 10^{-3}$ \\
$15-25$ & $(0.1 \pm 0.3) 10^{-6}$ \\
\hline
\end{tabular}

The main tool for this research is computational dosimetry using Monte-Carlo codes. Results are predominantly based on the MCNPX code package [13] and confirmation was sought from semi-independent simulation packages based on the GEANT4 framework [14], (see [15] for more information). Application code was written using the GEANT4 framework. Both codes, MCNPX and GEANT4, essentially use identical cross section libraries for the energetically relevent neutron interactions in matter (below $20 \mathrm{MeV}$ neutron energies); hence, they are referred to as semi-independent even though all other algorithms are different.

An indication of the shielding effect of various neutron absorber materials can be seen in Fig. 1. The boronated polyethylene (PE) shields perform best in terms of neutron number reduction for thin shields $(<5 \mathrm{~cm})$. After $5 \mathrm{~cm}$ thickness, the PE+Lithium shield excels. The hydrocarbons containing admixtures of neutron capture materials clearly dominate pure PE in terms of attenuation but it should be noted that this effect is due to thermal neutron numbers being significantly reduced and not due to an overall effect on the full neutron population. The cheapest shielding material, water, unfortunately also turns out to be the worst in performance. For the mixture hydrocarbon materials the cost factor is a non-negligible constraint, particularly for larger thicknesses.

However, the observable total neutron number alone is often not the most important for experiments interested in energy measurements. It appears that studies focussing on total neutron number have served, thus far, to define design criteria for shields. Here it is argued that the energy dependence of the penetrating flux is at least as important as number counts; in addition, build-up factors from neutron inelastic reactions either in terms of neutron multiplication or $\gamma$-production are other important factors to consider. 


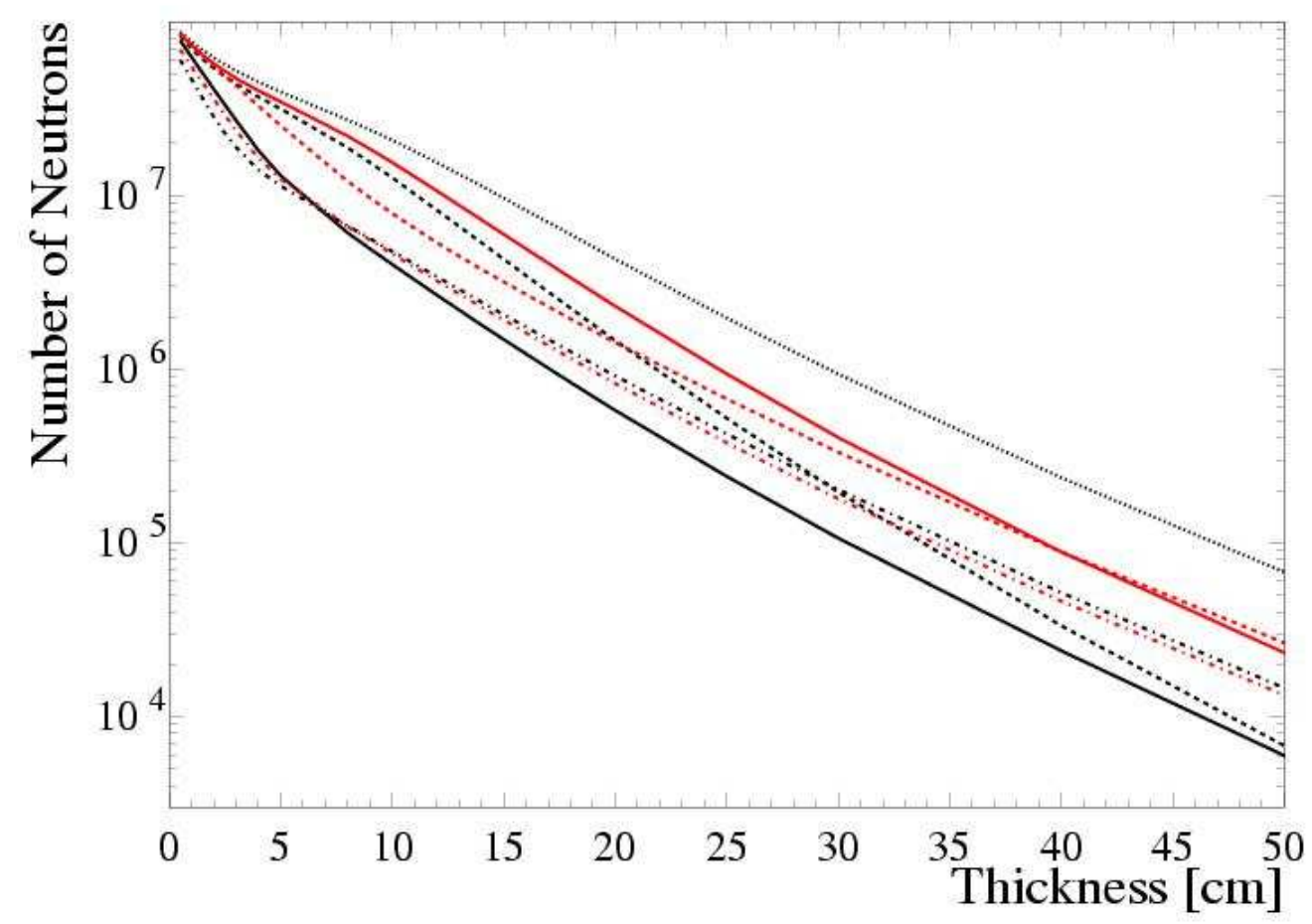

Fig. 1. Neutron shielding effects shown as a function of absorber thickness for various bulk materials. From top to bottom (at $15 \mathrm{~cm}$ thickness): Water (dotted), pure polyethylene (PE, solid grey), PE with bismuth (dashed), Premadex (grey dashed), PE with $30 \%$ boron content and $5 \%$ boron content (dash-dotted, B30\% in black, B5\% grey) and PE with lithium (solid).

Of particular interest for this study is the $(n, \gamma)$-reaction on metals and hydrocarbons and also the bulk material self-absorption effect. To investigate, bulk materials of copper, iron, lead, pure polyethylene and the mixtures $\mathrm{PE}+\mathrm{Li}$ and $\mathrm{PE}+$ Boron(30\% enriched) have been defined to have a $10 \mathrm{~cm}$ thickness and have been sliced into $4 \mathrm{~mm}$ pieces. Photon population number in each $4 \mathrm{~mm}$ slice was registered even though a pure neutron beam source (with Gran Sasso spectrum) was started. The results can be inspected in Fig. 2.

The $\gamma$-population per source neutron as a function of material thickness confirms the usual assertion that lead is a superior shielding material not only for $\gamma$-radiation but also due to its small reaction cross sections with neutrons. The overall $\gamma$-production from lead is far smaller than from all other metals in this study, reaching roughly $6 \%$ at maximum. Pure PE can go up to $20 \%$ but standard shielding metals like copper and iron reach well above plastics, almost to $50 \%$ in the case of copper. The hydrocarbon-based materials display a remarkable feature that should be bourne in mind when applying them to neutron shielding. Despite comparable performance with respect to neutron moderation and capture, there nevertheless is a huge difference in terms of 

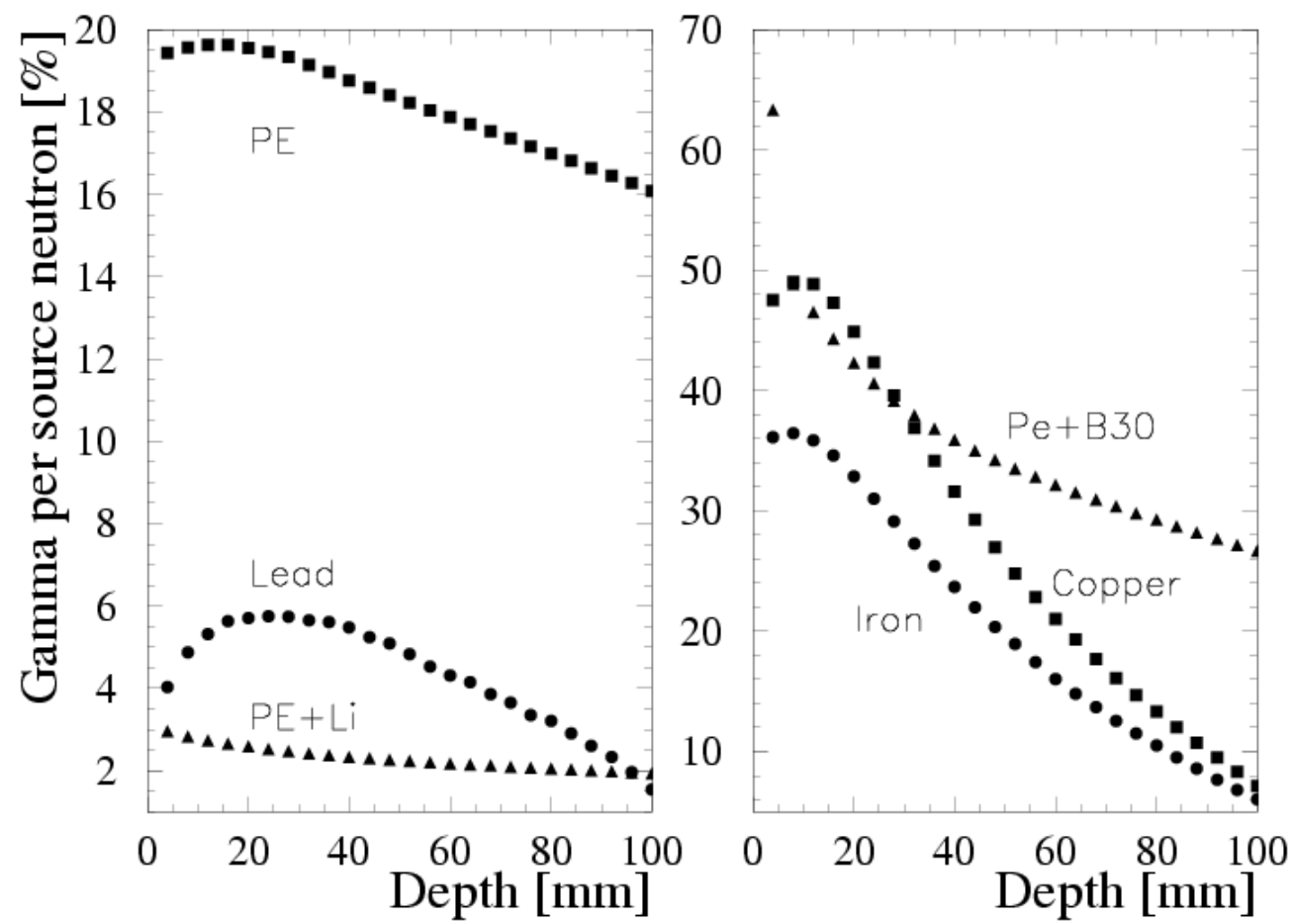

Fig. 2. $\gamma$-ray population per source neutron as a function of material thickness. A total depth of $10 \mathrm{~cm}$ has been divided into $4 \mathrm{~mm}$ slices. Shown is the gamma population, i.e. the number of photons present in each slice including actually produced photons and transported photons from other locations. As source, a pure neutron beam with Gran Sasso energy spectrum has been used. As bulk materials, pure PE, lead, PE+Lithium (all on the left panel), copper, iron and PE $+30 \%$ Boron (right panel) have been chosen.

photon production. The Boron admixture produces a vast amount of photons, particularly in the first slice, i.e. in the first $4 \mathrm{~mm}$. The lithium mixture shows very little photon production, in fact even fewer than lead, and singles itself out as a potential ideal capture material close to detectors.

Self-absorption of $\gamma$-rays can also be seen from this figure. PE and its mixtures hardly re-absorb their own $\gamma$-radiation, whereas the metals are more efficient. Iron produces a maximum number of photons around $36-37 \%$ compared to just $6 \%$ for lead. After $10 \mathrm{~cm}$ thickness, lead has reduced its value of gammas per source neutron below $2 \%$ but copper needs more than $10 \mathrm{~cm}$ to compete. These results for inelastic neutron reactions might be of interest for experiments having to shield $\gamma$-rays as well as neutrons.

The same simulations can also be used to quantify single material performance for pure neutron shielding. For this purpose, it is most revealing to study the energy-dependence of the neutron flux as it progresses through a given bulk material as a function of depth. This way, depletion of neutrons by absorption 


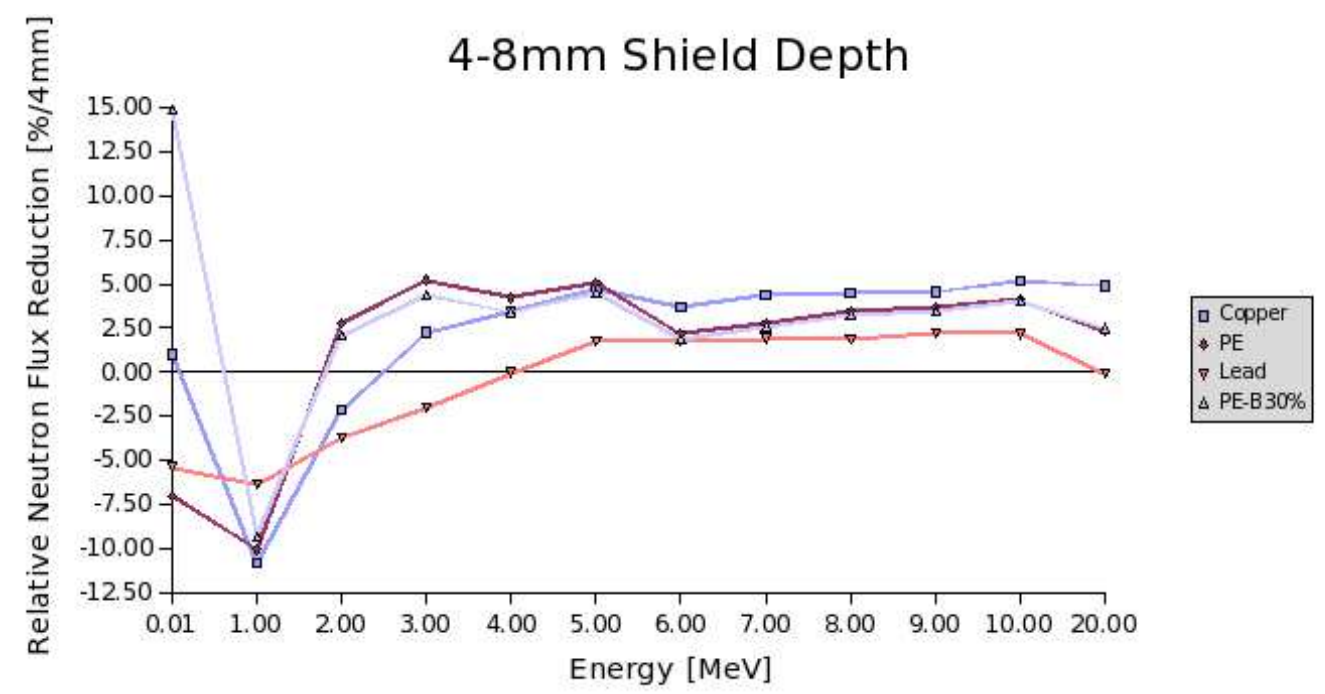

Fig. 3. Relative flux reduction per energy bin for copper, PE, lead and PE-B30\% as bulk shielding materials. A Gran Sasso neutron flux input spectrum is made to impinge on a $10 \mathrm{~cm}$ total thickness slab of material in the form of a point-like directed beam source. The result at a depth of $4-8 \mathrm{~mm}$ material thickness is shown. Lateral dimensions are large compared to the thickness. The neutron flux is tallied at the surface. The relative flux reductions result from flux changes through the surfaces. A reduction of flux for a given energy bin results in a positive value and flux enhancement in a negative value. The flux changes are all normalised to the minimum slab thickness of $4 \mathrm{~mm}$. The energy bin values contain flux up to the energy value, i.e. the first bin integrates flux from thermal energies up to $10 \mathrm{keV}$, then from $10 \mathrm{keV}$ up to $1 \mathrm{MeV}$ etc.

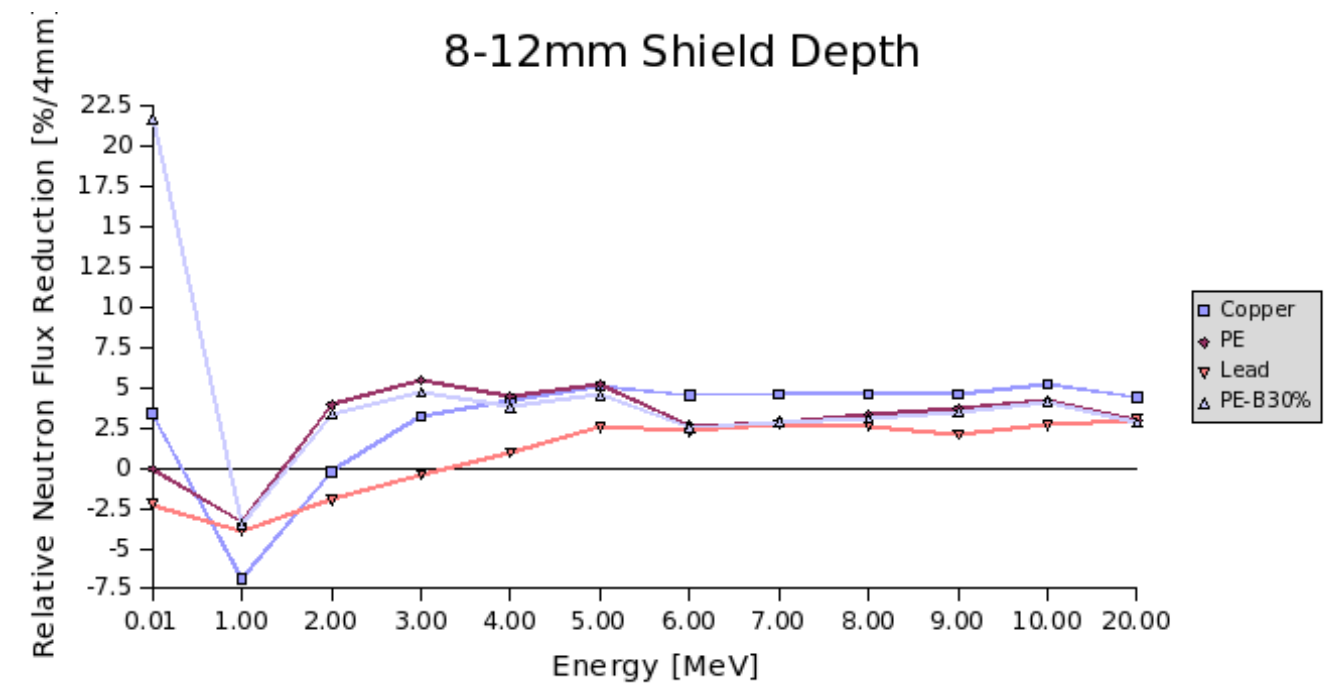

Fig. 4. As in figure 3 except the result shown is for a depth of $8-12 \mathrm{~mm}$.

and 'feeding' of low energy neutrons by moderation of higher energy ones can be demonstrated. 


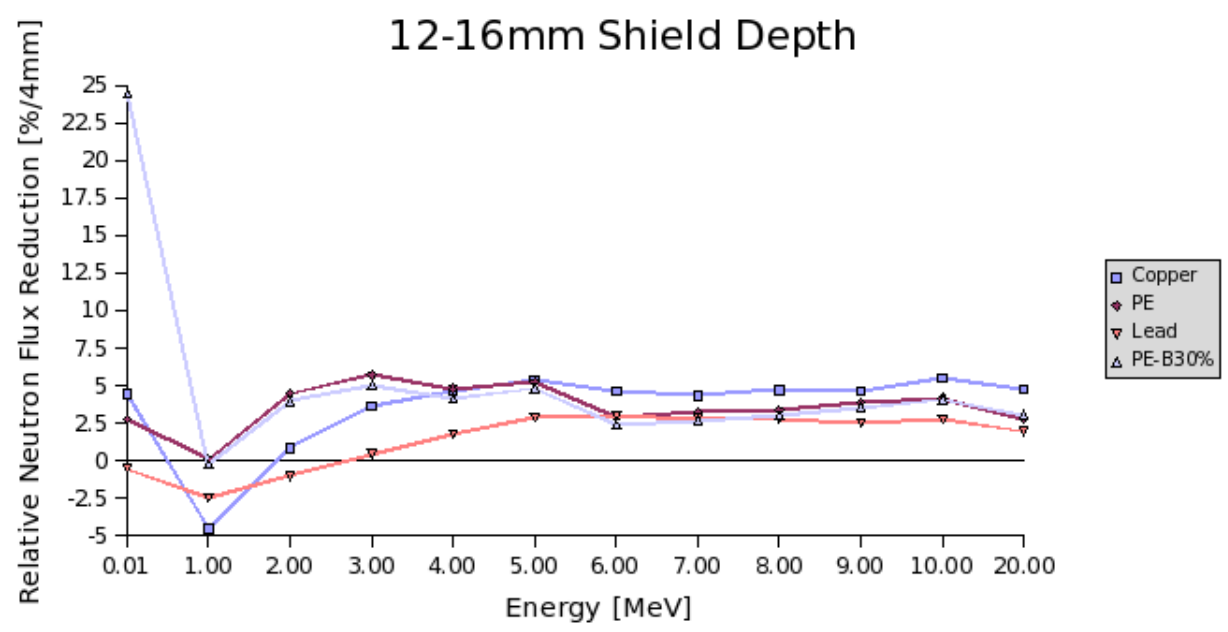

Fig. 5. As in figure 3 except the result shown is for a depth of $12-16 \mathrm{~mm}$
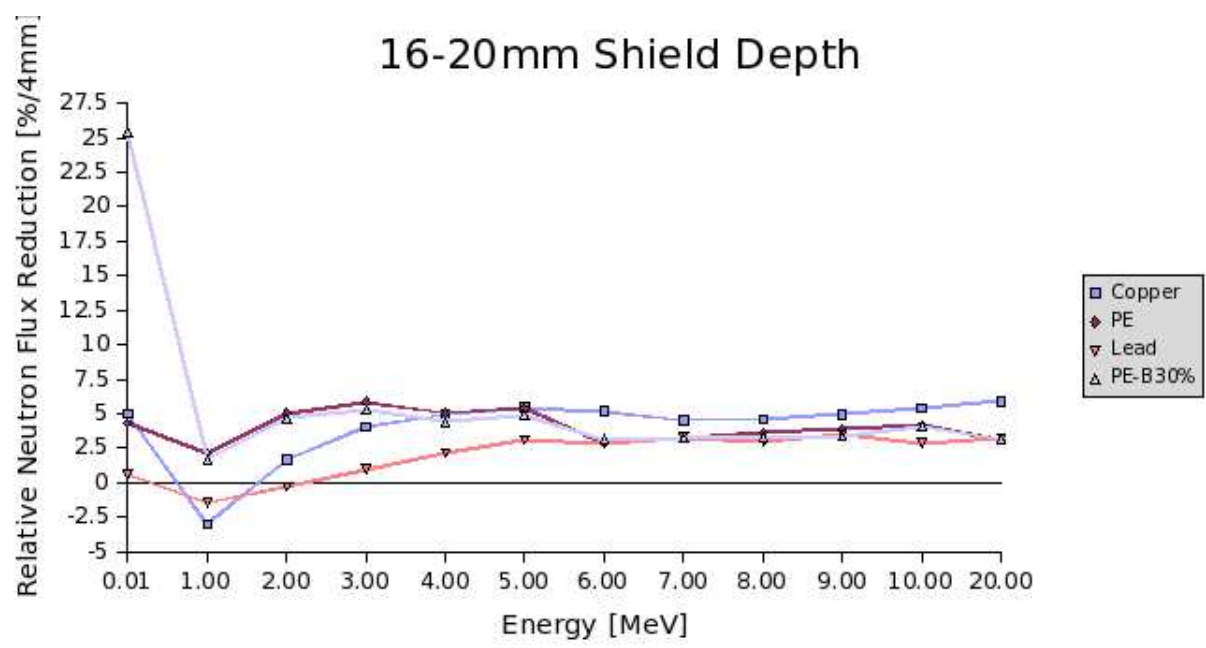

Fig. 6. As in figure 3 except the result shown is for a depth of $16-20 \mathrm{~mm}$

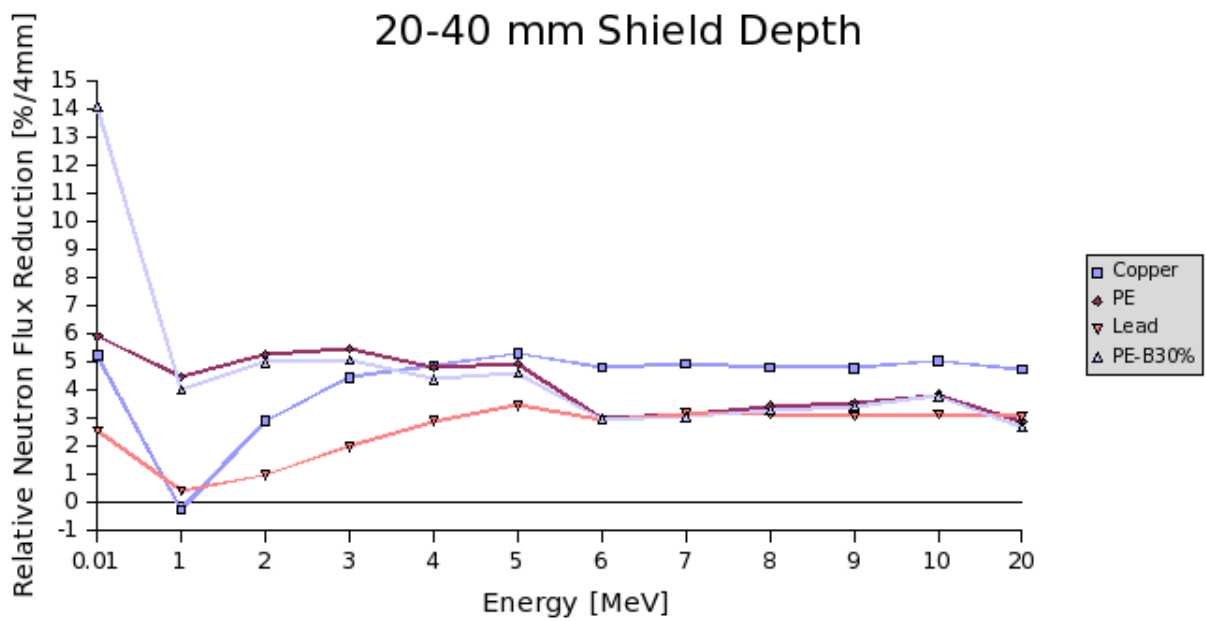

Fig. 7. As in figure 3 except the result shown is for a depth of $20-40 \mathrm{~mm}$ 


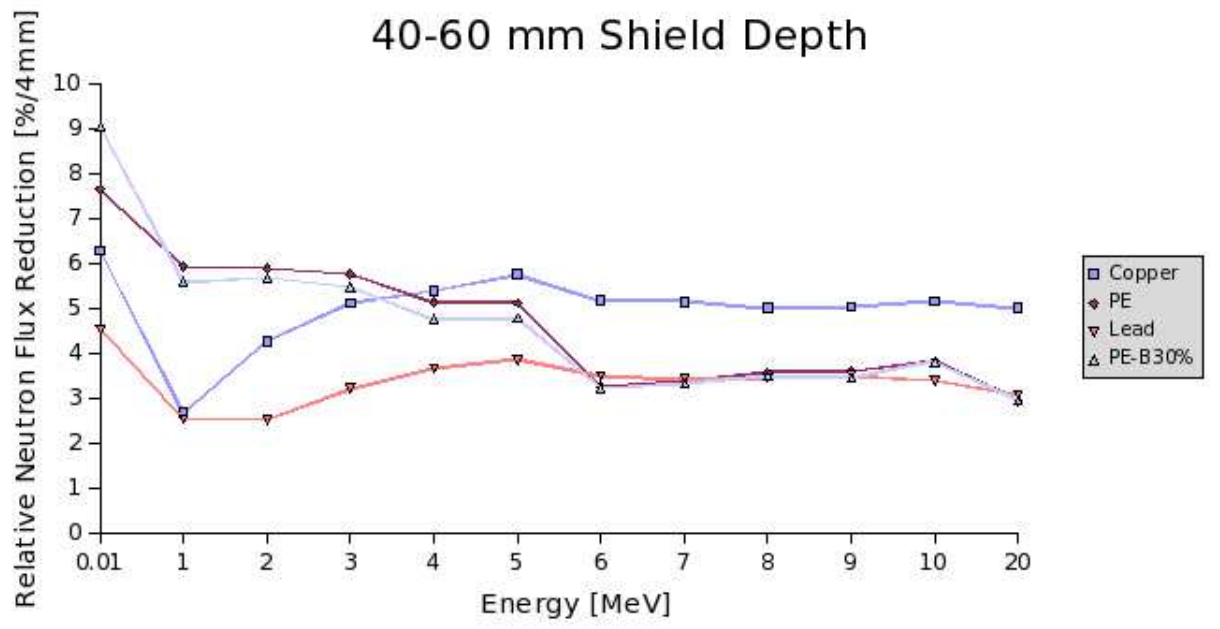

Fig. 8. As in figure 3 except the result shown is for a depth of $40-60 \mathrm{~mm}$

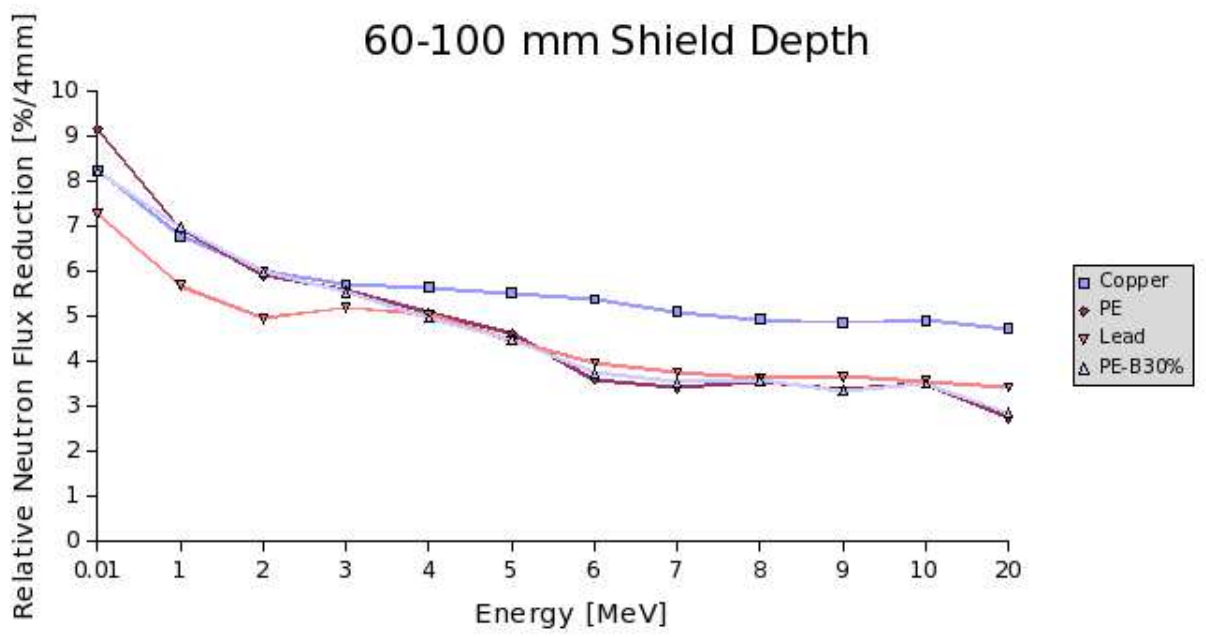

Fig. 9. As in figure 3 except the result shown is for a depth of $60-100 \mathrm{~mm}$

Figures 3 to 9 show the performance of the most relevant materials in radiation shields including pure polyethylene and a special neutron capture and moderator combination material, PE-boron (30\% B content). Each figure shows the reduction in neutron number flux as a function of energy for bulk material slices of $4 \mathrm{~mm}$. As before, a neutron beam with Gran Sasso energy spectrum is transported through a $10 \mathrm{~cm}$ thick wall of material. The charts show a positive $y$-value for the relative flux change between indicated surfaces (i.e. the case where neutron number is reduced) for the specified energy interval, and a negative value in the case where enhancement of neutron number takes place. The neutron flux is tracked for each $4 \mathrm{~mm}$ until a depth of $20 \mathrm{~mm}$ has been reached, then in steps of $20 \mathrm{~mm}$ up to a depth of $60 \mathrm{~mm}$ and finally, the last step to the exit-face of the wall amounts to $40 \mathrm{~mm}$.

Something to bear in mind when studying figures 3 to 9 is that the reduction in each $4 \mathrm{~mm}$ slice is as a percentage of the number of neutrons incident on that 
particular slice, rather than of the original number incident on the outermost layer. The figures must therefore be studied with care so that a reduction within a particular slice is not confused with an overall reduction in the original number incident on the outer surface of the slab of material. An example of where this may happen is in the $1 \mathrm{MeV}$ energy bin for copper or lead. Initially there is an overall increase in the number of neutrons in the outermost slices but eventually, at greater depths in to the material, neutron numbers are reduced. The neutrons must pass further through the slices before there are fewer neutrons in that energy bin than were intitially incident on the outermost slab.

Each of the materials presented display some unique features which can give important constraints on their usefulness as part of a neutron shield. Lead generally shows a relatively small influence on the neutron flux which can be seen from the values of reduction or enhancement, respectively, compared with all other materials presented. In addition, a comparatively large thickness of lead is necessary before the metal starts to reduce neutron flux over all energies. Sheets of lead with thickness below $2 \mathrm{~cm}$ will rather enhance neutron numbers, even at relatively high neutron energies below $2 \mathrm{MeV}$. The more common lead-brick with minimum thickness of about $5 \mathrm{~cm}$, however already reduces neutron fluxes significantly and provides a substantial self-shielding thickness against $(n, \gamma)$-radiation (see Fig. 2). Thick lead layers would even start to reduce efficiently a thermal neutron population.

Copper (as well as Iron, not shown here) as a neutron shielding material displays a remarkable thermal neutron capture cross section such that thin layers efficiently reduce a thermal neutron population. Nevertheless, a layer below $8 \mathrm{~mm}$ thickness would enhance the neutron flux between $1 \mathrm{MeV}$ and $2 \mathrm{MeV}$. The overall reduction is significantly stronger than lead for higher energies (5-6\% compared to roughly $3 \%$ ).

The classic neutron moderator, polyethylene, changes the neutron flux similarly to copper apart from the drastically different behaviour for low-energy neutrons. Another, more subtle difference can be inspected at the highest incoming energies, where the high density of copper results in stronger moderation of neutrons compared to PE. Below about $5 \mathrm{MeV}$, PE starts to scatter neutrons more effectively. Additionally for thicker slices, beyond $4 \mathrm{~cm}, \mathrm{PE}$ also absorbs such moderated neutrons more and more, favouring thick shield sizes for PE as a neutron shield material. Thin slices would inevitably lead to significantly enhanced neutron populations at low energies.

A 30\% enrichment of PE with natural boron as thermal neutron absorber and corresponding density increase, yields a significantly different picture compared to pure PE. A strong reduction for low energy neutrons can be seen for thin shield walls. At higher energies the shape of the reduction as function of 
Table 3

Suggested minimal thicknesses of bulk material. The last column indicates the reason. Neutron attenuation means thickness according to Figs. 3 to 9 , whereas $(n, \gamma)$ self-shielding comes from Fig. 2 and is defined as that thickness where photon production probability reaches initial (after $4 \mathrm{~mm}$ ) values again, i.e. after the maximum.

\begin{tabular}{lll}
\hline Material & Minimal thickness & criterium \\
\hline Lead & $20-40 \mathrm{~mm}$ & effective neutron attenuation \\
Lead & $70 \mathrm{~mm}$ & $(n, \gamma)$ self-shielding effective \\
Iron & $20-40 \mathrm{~mm}$ & effective neutron attenuation \\
Iron & $14 \mathrm{~mm}$ & $(n, \gamma)$ self-shielding effective \\
Copper & $40-60 \mathrm{~mm}$ & effective neutron attenuation \\
Copper & $16 \mathrm{~mm}$ & $(n, \gamma)$ self-shielding effective \\
PE & $16-20 \mathrm{~mm}$ & effective neutron attenuation \\
PE & $30 \mathrm{~mm}$ & $(n, \gamma)$ self-shielding effective \\
PE-Bi and PE-B & $12-16 \mathrm{~mm}$ & effective neutron attenuation \\
PE-Li & $8-12 \mathrm{~mm}$ & effective neutron attenuation
\end{tabular}

energy is practically identical to pure PE. Already thin slices of such enriched PE can significantly change the composition of a neutron population.

Now taking into account these pieces of information, one might start to compose a shield that most effectively attenuates neutrons and $\gamma$-rays under all circumstances. The minimal thicknesses of shielding materials taken from this section can be seen in Tab. 3 .

\section{Multilayer Shield Configurations}

Any neutron shield must be constructed such that higher energy neutrons are moderated as fast as possible and subsequently captured in order to significantly remove neutrons. An added complication for a shield design can be the accompanying $\gamma$-radiation from capture processes. Furthermore, a shield should be as compact and cost-effective as possible. Combining these constraints was the main purpose of this study.

One idea that was investigated, having established a suitable combination and configuration of materials, was testing small repeated blocks of layers, see Fig.10, to see if it offers a greater reduction in total neutrons and photons compared to a single large block of equal total thickness. The reasoning behind this was that the blocks of layers may yield approximately exponential 


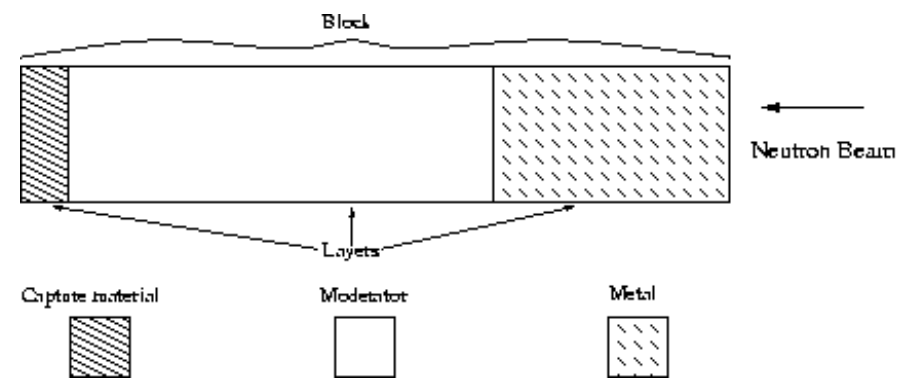

Fig. 10. Diagram of a single block made up of layers of a moderator, capture material and a metal.

attenuation of incoming flux at each block. In principle, this idea cannot be optimal since after the first block, the flux has changed in shape considerably such that a repetition might not be the best way to remove incoming flux at that position. It was agreed that a multilayer configuration was worth testing nevertheless.

Starting with a minimum layer depth for all realistically available materials, see Tab. 3, a minimal block unit of layers was constructed after all possible permutations (ordered with respect to neutron beam arrival direction) of up to four materials had been put to test.

The chosen method for the comparison of various configurations was to first fix the single block depth to $15 \mathrm{~cm}$, leaving enough space to accommodate thicknesses of single materials in the range of minimal requirements. Second, a layer configuration is arranged such that moderation and capture should happen in series, see below and [19]. Capture materials in front of moderators have been excluded from this study after preliminary simulations showed significantly inferior performance as expected. The remaining possible variations comprise the placement of high-density materials for either photon capture or neutron capture and moderation or even both. Three sets of configurations have been tested, either a metal behind or in front of a moderator+capture structure or both. Best results for neutron attenuation were obtained for a metal in front of the moderator+capture material, see Fig.10.

An $80 \mathrm{~cm}$ total shield thickness allowed 3 blocks of these layers to be sandwiched between $15 \mathrm{~cm}$ lead and $20 \mathrm{~cm}$ pure PE for simulations, see discussion on this 'clamp' below. The 3-block arangement was chosen as arbitrary. In all of the following tests, $10^{8}$ particles with a Gran Sasso spectrum were simulated.

Using lead as the metal, PE-Bi as the moderator and PE-Li as the capture material as an initial best guess, tests were performed to optimise the ratio of one material thickness to another. In total, 25 ratio configurations were tested and a 3:8:4 configuration (3cm PE-Li, 8cm PE-Bi, 4cm Lead) was seen to be best for an overall reduction of both neutrons and gammas, although this was not best for neutron or gamma reduction alone, see Fig.11. A 2:12:1 


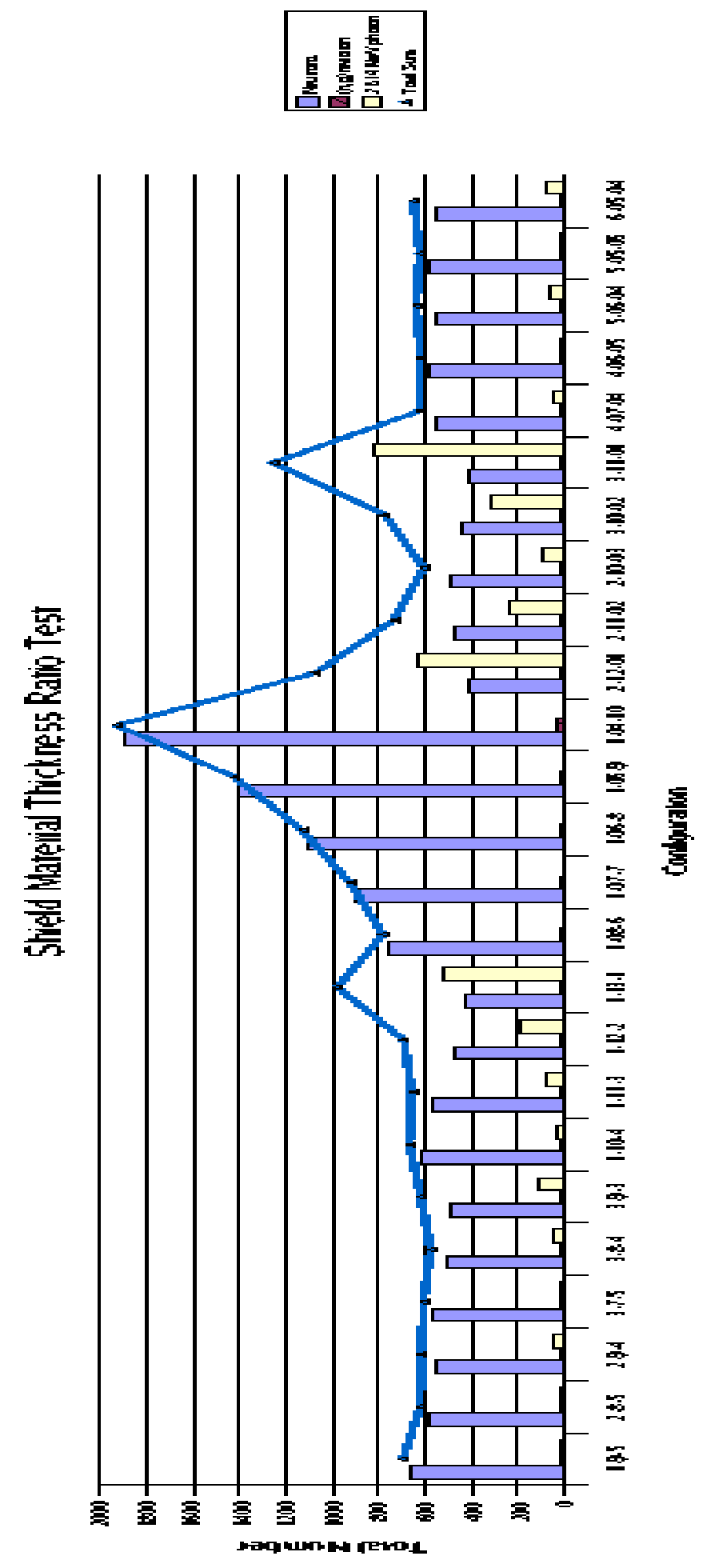

Fig. 11. 25 different ratio configurations of material thickness were tested using Lead as the metal, PE-Bi as the moderator and PE-Li as the capture material. The best configuration for overall particle reduction is shown to be $3: 8: 4$ (3cm PE-Li, $8 \mathrm{~cm}$ PE-Bi, 4cm Lead). 


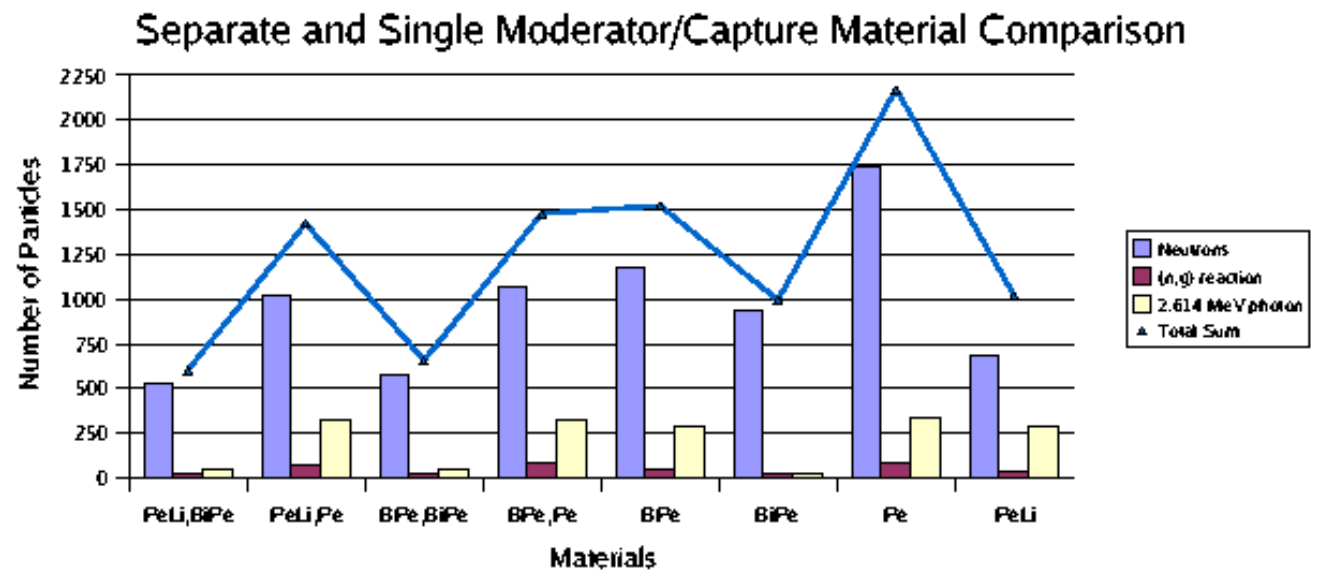

Fig. 12. Comparison between single capture/moderator materials and a separate moderator then capture structure. Each structure follows the 3:8:4 pattern, as previously used, with lead as the metal in each case. In the case where a single material is used, the total thickness of moderator and capture material is taken i.e. $11 \mathrm{~cm}$.

configuration was best for neutron reduction and any configuration with $9 \mathrm{~cm}$ of lead or more removed all gammas (excluding those from $(\mathrm{n}, \gamma)$ reactions).

The reader should bear in mind that the moderator material will follow the pattern presented in Fig.1 for neutron reduction versus thickness but the capture material will behave differently due to the fact the moderator has had an effect prior to reaching the capture material surface. In general, a $1 \mathrm{~cm}$ increase in PE-Li at the expense of $1 \mathrm{~cm}$ in $\mathrm{PE}-\mathrm{Bi}$ will have a greater effect on number reduction due to the small amount of PE-Li simulated in comparison to PE-Bi. A small amount of PE-Li is tested as it is not sold in large blocks, although it is generally cheaper than PE-Bi. It appears that $4-5 \mathrm{~cm}$ of lead is optimal at these scales as further thickness increase has little more effect on gamma number and $(\mathrm{n}, \gamma)$ reactions have a minimal contribution to total numbers so may be ignored.

Following the material ratio testing, further simulations were run in order to find the best combination of materials. The metal was exchanged between lead and iron for comparison whilst the moderator was swapped between PE and Bi-PE. Results show that Bi-PE is the better moderator and that lead is better in overall particle reduction. PE-Li was compared to PE-B and was found to out-perform as a capture material. As a single material, lead is more effective than iron at gamma attenuation but iron is more suited to neutron reduction.

Another question that was addressed was whether it is better to have a separate moderator-capture configuration or to combine the two tasks using a single material. The results of simulations show a definite argument towards 
a separate moderator and capture material for overall particle reduction, see Fig. 12. The best material combination was using PE-Bi for the moderator and PE-Li for the capture material.

The next stage in the testing was to then find out whether a single large layered block would achieve better or worse results than repeated smaller blocks of the 3:8:4 configuration, both sandwiched between $15 \mathrm{~cm}$ lead (inside) and $20 \mathrm{~cm}$ pure PE (outside). This was done by fixing the total shield thickness to $80 \mathrm{~cm}$, keeping the 3:8:4 ratio constant and adjusting the number of blocks in the sandwich by varying the block size proportionally. The outcome of this can be seen in Fig. 13.

Results show that a repetition of two blocks reduced the total number of particles passing through the shield, although not significantly, see Fig 13. There was very little difference between a single elongated block and two smaller blocks with the same material thickness ratio; the results for the two blocks were within percentage errors. Further repetitions of blocks had a detrimental effect.

A subsequent test to see whether an outermost pure moderator layer would enhance the multilayer shield, showed that this improves overall performance considerably due to the initial softening of any incoming neutron spectrum. Both default layers (introduced as educated guess structures initially), bracketing the multilayer structure, do not need to be thick. An additional $5 \mathrm{~cm}$ inner lead (should be low in ${ }^{210} \mathrm{~Pb}$ as usual, see [3]) would already remove almost the entire photon flux from any neutron interaction. The softening moderator outside can be a $10-20 \mathrm{~cm}$ pure PE layer, representing a compromise in moderation efficiency and in cost and weight.

Experiments that rely on removing environmental photon flux entirely should always aim at a total of $30 \mathrm{~cm}$ lead for the $2.614 \mathrm{MeV}$ line from the Thorium chain. Therefore it might be necessary to increase the inner lead layer to a total of $15 \mathrm{~cm}$.

Considering that this proposed multilayer structure is clamped between a very conventional shield configuration (PE outside, lead inside) the question arises whether it is necessary at all. This topic is discussed in the next section.

\section{Comparison with Standard Shields}

In order to gauge how well the shields in the previous section (ML1-6) would perform compared to a selection of six existing shielding designs (Std1-Std6), simulations using MCNPX were run, see Fig. 14. A Gran Sasso flux beam 


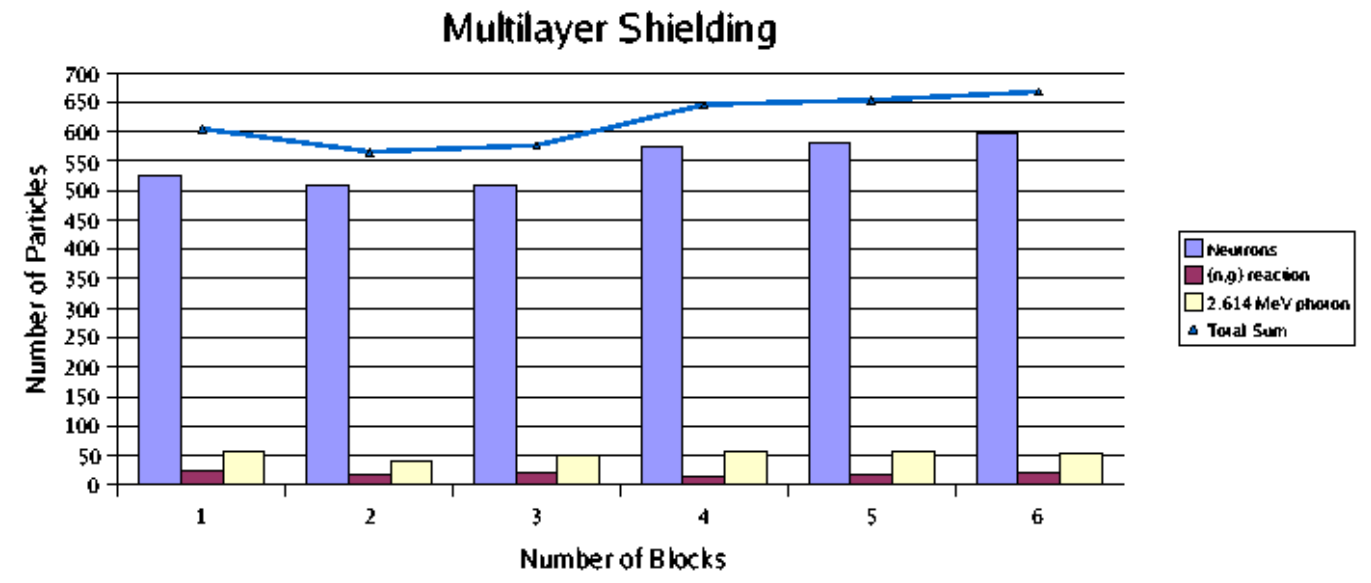

Fig. 13. Comparison of results for varying the size and therefore the number of blocks sandwiched between $15 \mathrm{~cm}$ lead and $20 \mathrm{~cm}$ PE giving a total shield thickness of $80 \mathrm{~cm}$. The ratio of materials in each block is kept at 3:8:4 (PE-Li:PE-Bi:lead).

consisting of $10^{8}$ neutrons was started as well as a mono-energetic (2614.5 $\mathrm{keV}$ ) gamma-ray beam source. The performance of each shield was based on how many neutrons and gammas would leave the exit face of the shielding. For all configurations a total thickness of $80 \mathrm{~cm}$ has been set, determined by the proposed configurations.

The pure PE shielding (Std1) is considered in $[8,9]$ as a viable shielding option although with a thinner total depth. The main difference between Std2,3 and Std5,6 is merely the lead thickness. Std2 and Std5, respectively, have the classic PE-lead configuration, where it should be pointed out that $30 \mathrm{~cm}$ lead is not unusual but $50 \mathrm{~cm} \mathrm{PE}$ is considered to be a very massive moderator [3]. The configurations Std3 and Std6 are more recent actual shields employed in dark matter experiments but with different, thinner layer depths. Here the inner moderator is considered to be an effective method to moderate neutrons originating from the shielding material itself, in particular from the lead layer by neutron spallation reactions. The Std 4 shield is another conventional shield configuration, seen in dark matter as well as double-beta searches. The copper layer in thinner form would typically represent an inner lining to remove lead X-rays [3].

As discussed in the previous section, the proposed shields contain a number of consecutive blocks of multilayers, clamped between a conventional Lead-PE structure (ML1-6 in Fig.14). The results of the simulations can be seen in Fig.15. It can be seen that even the poorest of performers from ML1-6 still exceeds performances from all of the existing shields that were tested.

The standard shields exhibit some interesting features. Note that the pure neutron fluxes of Std1,3 and 6 are comparable although moderator thicknesses 


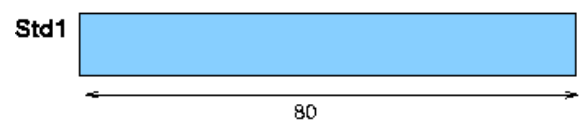

Std2

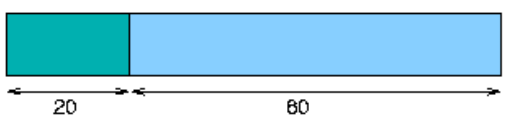

Std3

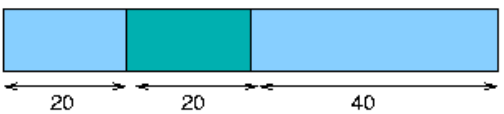

Std4

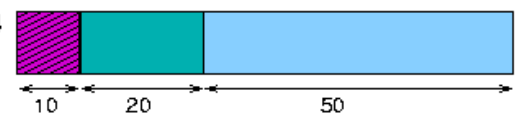

Std5

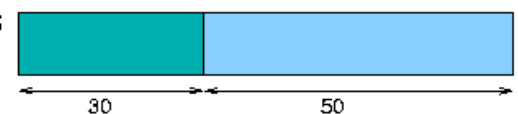

Std6

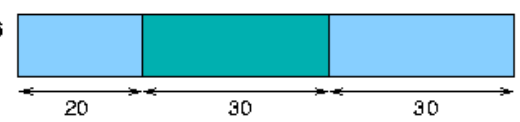

ML1

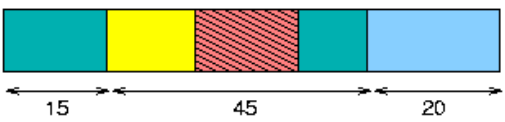

ML2

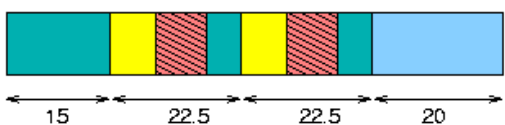

ML3

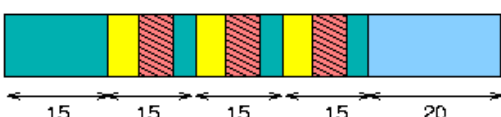

ML4

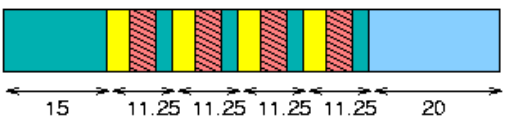

ML5

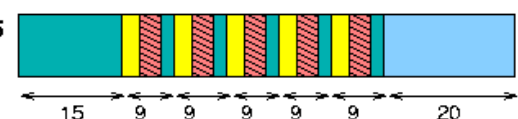

ML6

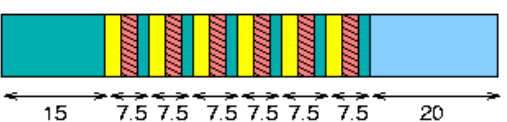

KEY

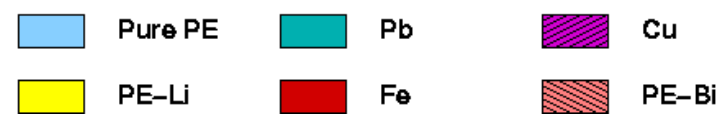

Fig. 14. Shielding configurations simulated for comparison of standard shields (label: Std) to proposed multilayer shields (label: ML). Each of the ML shields keep the thickness ratio of PE-Li:PE-Bi:Pb to 3:8:4 as this was shown to be the best configuration from the simulations. For discussion, see text.

vary from 80 to $50 \mathrm{~cm}$ total. Disturbing the effect of pure PE on neutrons by implementation of lead has a favourable consequence on neutron attenuation (compare Std2,3 and Std5,6). However, the effect on the photon population is opposite. The build-up factor and $(n, \gamma)$-reactions increase the total considerably and strongly favour lead as last layer. Replacing the last $10 \mathrm{~cm}$ of lead with copper (Std5 to 4) also shows a non-negligible effect on the photon population.

Finally, it might be interesting to note that the multilayer shields exhibit similarly strong attenuation of neutrons to the pure PE shield. The added multilayer advantage of more than four orders of magnitude suppression of photon flux compared with the pure PE shield might be of interest, even for quasi $\gamma$-ray insensitive detectors.

\section{Conclusions}

A survey of available radiation shielding materials has been presented. An effective configuration for combined neutron, photon attenuation was derived and compared to existing configurations for radiation shields in underground 


\section{Shield Configuration Comparison}

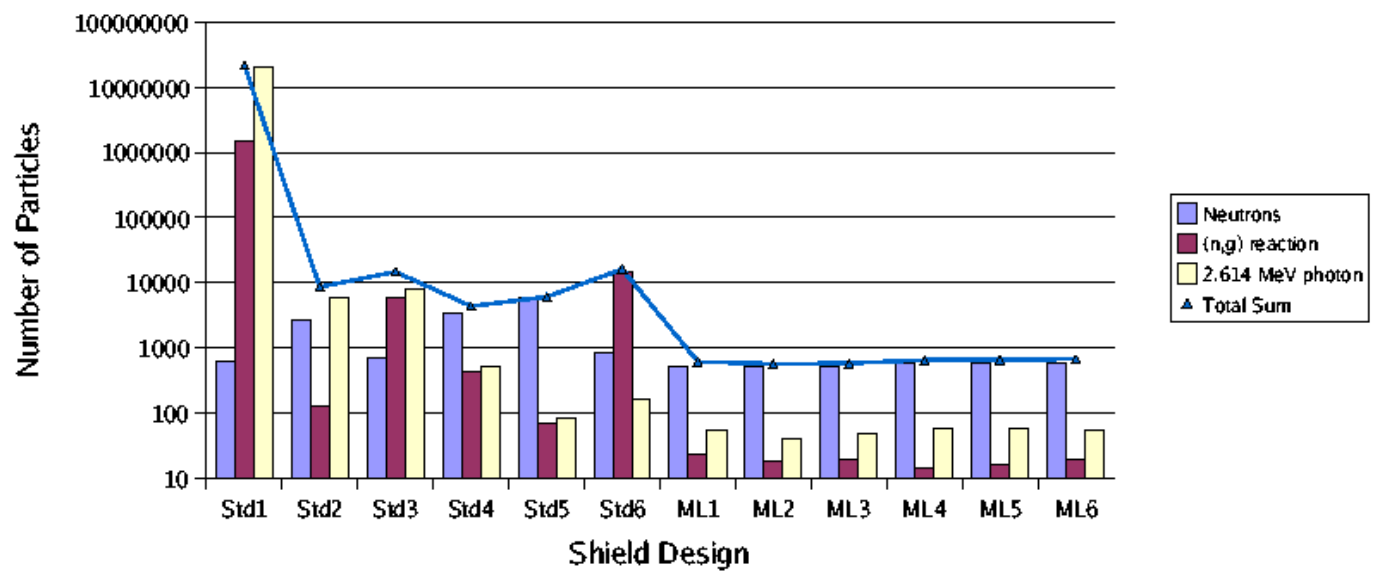

Fig. 15. Shield configuration comparison for six standard shields, labeled 'Std' and six multilayer shields, labeled 'ML'. The configurations can be seen in Fig. 14. Shown here is the total number of either neutrons or photons tallied at the exit surface of the respective shield configurations. The first bar shows neutron numbers and the second photon numbers from $(n, \gamma)$-reactions. Those result from a Gran Sasso neutron flux beam source, starting $10^{8}$ neutrons. The third shows photon numbers from a monoenergetic $(2614.5 \mathrm{keV}) \gamma$-ray beam source, starting $10^{8}$ photons. The line indicates values for the total sum of neutrons and photons traversing the shield.

nuclear and particle physics experiments. For a total background reduction in this kind of experiment it is proposed that multilayer shields composed of a metal, moderator and capture material, as in Fig.10, are used. The multilayer structure turns out to be more efficient in dealing with neutron flux evolution. Thermal neutron absorbers have been shown to be very efficient already when used as thin sheets rather than as significantly more expensive bulk material. For pure moderation purposes, simple PE is sufficient. Metals are necessarily present for $\gamma$-ray attenuation but have been shown to be interesting also for their influence on neutron fluxes.

Finally, it should be pointed out that this study was concerned with passive shielding only. The neglected background contributions due to muons, even in underground laboratories, are best dealt with using active shielding components such as plastic scintillator panels mounted outside the shielding (see e.g. $[3,18])$. For COBRA, improvements on the passive shielding design have resulted in the development of an active element which could be mounted as an inner layer. An active element in this position can act as a veto for any last remaining external background. Further studies examining the minimal passive shield configurations for COBRA, subject to cost constraints, are ongoing. 


\section{References}

[1] K. Zuber, Phys. Lett B 519 (2001) 1

[2] Conference Proceedings of the ICRS-10 and RPS-2004 conference series, Madeira, May 9-14, 2004, to be published in Rad. Protection Dosimetry (2005); http://www.itn.mces.pt/ICRS-RPS/

[3] G. Heusser, Annu. Rev. Nucl. Part. Sci. 45 (1995) 543

[4] Y. Harima, Radiat. Phys. Chem. 41 (1993) 631

[5] G. Chardin, to be published in "Cryogenic Particle Detection", ed. Ch. Enss, Springer, Heidelberg, 2005; Preprint archive (http://arxiv.org/) astro-ph/0411503

[6] K. Shizuma et al., Nucl. Instr. Meth. B 66 (1992) 459

[7] C.A. Bisselle, A. Karam and J.A. Wethington, Int. J. Appl. Radiat. Isotopes 15 (1964) 529

[8] P.F. Smith et al., Astropart. Phys. 22 (2005) 409

[9] M.J. Carson, et al., Nucl. Instr. Meth. A 546 (2005) 509

[10] H. Wulandari et al., Preprint archive (http://arxiv.org/) hep-ex/0401032

[11] P. Belli et al., Nuov. Cim. 101A (1989) 959

[12] H. Wulandari et al., Astropart. Phys. 22 (2004) 313

[13] MCNPX User's Manual (2002), Los Alamos National Lab. publication LA-CP02-408, version 2.4.0; http://mcnpx.lanl.gov/

[14] GEANT4 Collaboration, Nucl. Instr. and Meth. A 506 (2003) 250

[15] R. Lemrani et al., Nucl. Instr. and Meth. A560 (2006) 454

[16] F. Lei et al., IEEE Trans. Nucl. Sci. 49 (2002) 2788

[17] G.F. Knoll, Radiation Detection and Measurement, Wiley, New York, 3rd ed. (2000)

[18] T. Schroettner, M. Schwaiger and P. Kindl, Appl. Rad. and Isotopes 61 (2004) 133

[19] J.Hong, W.W. Craig, C.J. Hailey, Nucl. Instr. Meth. A 452 (2000) 192 - 204 